\title{
Über Bleichromat.
}

Von Max Gröger.

Für die unlängst veröffentlichte Arbeit über die jodometrische Bestimmung von Chromsäure in Bleichromaten ${ }^{1}$ ) wurde vollkommen reines normales Bleichromat benötigt und versucht dieses durch Fällung gleicher Volumen genau äquivalentnormaler Lösungen von Bleiacetat und Kaliumchromat zu gewinnen. Da stellte sich das überraschende Ergebnis heraus, daB unter diesen Umständen nicht die äquivalenten Mengen $\mathrm{PbO}$ und $\mathrm{CrO}_{3}$ in den Niederschlag gehen, sondern eine beträchtliche Menge Blei in Lösung bleibt. Dies war die Veranlassung zu den im Nachfolgenden beschriebenen Versuchen über die Fällung von Bleiacetatlösungen mit Alkalichromaten.

Zur Verwendung kamen eine klare Bleiacetatlösung, von der $10 \mathrm{ccm}$ bei der gewichtsanalytischen Gehaltsbestimmung $1.5158 \mathrm{~g}$ $\mathrm{PbSO}_{4}$ ergaben und Lösungen von reinem Kalium-, Natrium- oder Ammoniumchromat, die nach jodometrischer Bestimmung in $1 \mathrm{l} 50 \mathrm{~g}$ $\mathrm{CrO}_{3}$ enthielten. Wenn im folgenden der Kürze halber nur von Bleilösung und Chromatlösung gesprochen wird, so sind stets diese Normallösungen gemeint.

LäBt man in die Bleilösung unter Umschwenken ein gleiches Volumen der Kaliumchromatlösung zutropfen, so entsteht ein hellgelber Niederschlag und eine farblose bleihaltige Lösung. Auch nach langer Berührung mit der Mutterlauge bleibt das Aussehen des Niederschlages unverändert. Bei mikroskopischer Untersuchung erweist er sich als aus sehr kleinen rundlichen Körnchen bestehend. Wird der Niederschlag durch ein gewöhnliches Papierfilter abfiltriert und darauf mit Wasser ausgewaschen, so erhält man zunächst ein klares farbloses bleihältiges Filtrat, sobald aber die Bleilösung weggewaschen ist, läuft die Flüssigkeit trüb durch das Filter, was auf eine kolloide Beschaffenheit des Niederschlags hinweist. Fängt man das von gelöstem Bleiacetat freie durch einen feinverteilten hellgelben Niederschlag getrübte Filtrat gesondert auf, so setzt sich dieser erst nach tagelangem Stehen vollständig ab, darüber steht dann eine

$\left.{ }^{1}\right)$ Z. anory. u. ally. Chem. 108 (1919), 267. 
klare gelbe Lösung, die beim Eindampfen Kaliumchromat hinterläBt. Der ursprüngliche Niederschlag ist somit kaliumhältig.und wird durch Wasser zersetzt.

Um seine Zusammensetzung zu ermitteln, wurde ein Niederschlag aus $20 \mathrm{ccm}$ der Bleilösung und $20 \mathrm{ccm}$ der Kaliumchromatlösung, nachdem er längere Zeit mit der Mutterlauge in Berührung geblieben war, vor der Saugpumpe abfiltriert und mit Alkohol andauernd ausgewaschen. Obwohl der Eindampfrückstand der wässerigen Mutterlauge, der nur Kalium- und Bleiacetat enthielt, in Alkohol leicht löslich, demnach zu erwarten war, daß sich die dem Niederschlag anhaftende Mutterlauge durch Alkohol leicht verdrängen lieBe, muBte das Auswaschen damit sehr oft wiederholt werden, bis endlich ein bleifreies Filtrat erhalten wurde. Bei dieser Behandlung behielt der. Niederschlag seine hellgelbe Färbung und sein mikroskopisches Aussehen. Die Analyse des bei $120^{\circ}$ getrockneten Niederschlags ergab:

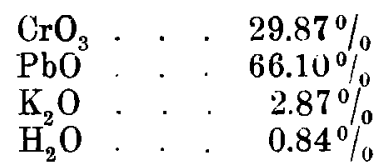

Daraus berechnet sich das Molekularverhältnis $\mathrm{CrO}_{3}: \mathrm{PbO}: \mathrm{K}_{2} \mathrm{O}$ $=1: 0.992: 0.102$. Nimmt man an, dab das Kalium als normales Chromat im Niederschlag gebunden ist, so bleibt als Rest ein Bleichromat mit dem Molekularverhältnis $\mathrm{CrO}_{3}: \mathrm{PbO}=1: 1.104$, somit ein basisches Salz.

Wird bei der Fällung der Bleilösung nicht das gleiche Volumen der Kaliumchromatlösung, sondern ein wenig mehr verwendet, so entsteht zwar zuerst wieder der hellgelbe Niederschlag, geht aber bald in einen viel voluminöseren orangegelben kristallinischen über, der unter dem Mikroskop sich aus dünnen kurzen Nädelchen bestehend erweist. Eir läBt sich gut filtrieren und gibt beim Auswaschen mit kaltem Wasser, wenn auch die anhaftende Mutterlauge schon längst verdrängt ist, immer noch kleine Mengen Kaliumchromat an dieses ab.

Zur Ermittlung der ursprünglichen Znsammensetzung wurde deshalb der kristallinisch gewordene Niederschlag aus $20 \mathrm{ccm}$ der Kaliumchromatlösung und $19 \mathrm{ccm}$ der Bleilösung anf dem Saugfilter durch Auswaschen mit Alkohol von Kaliumacetat befreit, dann getrocknet und analysiert. $\mathrm{Er}$ enthielt:

$$
\begin{aligned}
& \mathrm{CrO}_{3} \cdot \text {. } 31.50 \% \\
& \mathrm{PbO}^{3} . \quad .66 .99 \% \\
& \mathrm{~K}_{2} \mathrm{O} \text {. . } \quad 1.44 \%
\end{aligned}
$$


Daraus bereehnet sich das Molekularverhältnis $\mathrm{CrO}_{3}: \mathrm{PbO}: \mathrm{K}_{2} \mathrm{O}$ $=1: 0.953: 0.049$. Ist das Kalium als normales Chromat im Niederschlag, so bleibt als Rest ein Bleichromat mit dem Molekulirverhältnis $\mathrm{CrO}_{3}: \mathrm{PbO}=1: 1.002$, somit ein normales Salz. Das Kaliumchromat stammt zum Teil aus der dem Niederschlag anhaftenden Mutterlauge, zum gröBeren Teil aber ist es an Bleichromat gebunden.

Dadurch, dab beim Vermischen gleicher Volume der Blei- und Chromatlösung ein Teil des Kaliumchromats von dem zuerst ausfallenden Bleichromat gebunden wird, erklärt sich, daB ein Teil des Bleiacetats in der Mutterlauge verbleibt. Bei einer Fällung von $10 \mathrm{ccm}$ Bleilösung mit $10 \mathrm{ccm}$ Kaliumchromatlösung wurden $17.4 \mathrm{ccm}$ Mutterlauge abgesaugt, die bei der gewichtsanalytischen Bestimmung $0.0715 \mathrm{~g} \mathrm{PbSO}_{4}$ ergaben, entspechend $0.47 \mathrm{~cm}$ der Bleilösung. S0mit blieben etwa $5 \%$ der verwendeten Bleilösung im Überschub.

Der BleiüberschuB bewirkt auch, daB das ausfallende Bleichromat basisch wird, denn sowohl das hellgelbe amorphe, als auch das orangegelbe kristallinische Bleichromat nimmt aus Bleiacetat Bleioxyd auf. Bei Anwendung eines groBen Überschusses der Bleilösung gelingt es den Niederschlag vollständig in das orangerote Chromat $\mathrm{PbO} . \mathrm{PbCrO}_{4}$ überzuführen.

Um dies nacbzuweisen wurden in eine Porzellanschale mit $40 \mathrm{ccm}$ der Bleilösung $10 \mathrm{ccm}$ der Kaliumchromatlösung eingetroptt und nach gleichförmiger Verteilung des hellgelben Niederschlags unter häufigem Umrühren auf kochendem Wasserbade bis zur Trockene verdamptt. Es entwich dabei Essigsäure. Der Trockenrückstand mit kachendem Wasser ausgezogen hinterlieb einen orangeroten Niederschlag, der auf ein Filter gebracht und durch Auswaschen mit heißem Wasser von der auhaftenden Kalium- und Bleiacetat enthaltenden Lösung befreit wurde. Bei der Analyse des bei $120^{\circ}$ getrockneten Niederschlags wurden gefunden:

$$
\begin{aligned}
& \mathrm{CrO}_{3} \cdot-\quad 18.20 \% \\
& \mathrm{PbO}^{3} \text {. . } 81.55 \% \text {, }
\end{aligned}
$$

entsprechend dem Molekularverhältnis $\mathrm{CrO}_{3}: \mathrm{PbO}=1: 2.008$.

Fällt man die Bleilösung mit einem kleinen ÜberschuB der Chromatlösung und setzt erst dann, wenn der Niederschlag kristal-

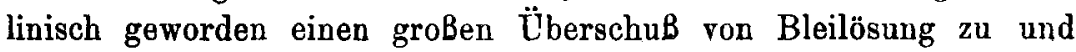
dampft ein, so geht die Bildung dieses basischen Bleichromats viel langsamer vor sich, man muB den Eindampfrückstand wiederholt mit Wasser aufnehmen und neuerlich eindampten um den orange- 
gelben Niederschlag völlig in den orangeroten überzuführen. Das amorphe Chromat ist also weit reaktionsfähiger als das kristallinische.

$\mathrm{DaB}$ der hellgelbe Niederschlag trotz der Anwendung eines Überschusses von Bleilösung bei seiner Fällung kalihältig ist, erklärt sich dadurch, daB eine Lösung von Kaliumacetat auf Bleichromat einwirkt, in der Kälte nur wenig, viel stärker beim Erhitzen. Es geht dabei Bleiacetat in Lösung und Kaliumchromat in den Niederschlag. Wird reines zu Pulver zerriebenes Bleichromat mit einem ÜberschuB von Kaliumacetatlösung auf dem Wasserbade eingedampft, so schwillt es zunächst auf, färbt sich orange und erst wenn die Lösung ganz eingedickt ist zitronengelb. Wird der erkaltete Eindampfrückstand mit kaltem Wasser aufgenommen, filtriert und ausgewaschen, so läuft zuerst eine farblose klare bleihältige Lösung durch das Filter, dann tritt eine geringe gelbliche Trübung auf, bald aber wieder werden die Waschwässer ganz klar, sind aber dann gelblich gefärbt. Daraus geht hervor, dab der Niederschlag ein Kaliumbleichromat euthält, welches von Bleiacetat nicht angegriffen, aber durch reines Wasser langsam zersetzt wird.

$\mathrm{Om}$ die Zusammensetzung dieser Verbindung zu bestimmen wurden $2 \mathrm{~g} \mathrm{PbCrO}_{4}$ mit $30 \mathrm{ccm} \mathrm{n}-\mathrm{KC}_{2} \mathrm{H}_{3} \mathrm{O}_{2}$ in der eben beschriebenen Weise behandelt. Das Auswaschen wurde, sobald die Waschwässer bleifrei geworden, unterbrochen. Die Analyse des bei $120^{\circ}$ getrockneten schön gelben Niederschlags ergab:

$$
\begin{aligned}
& \mathrm{CrO}_{3} \text {. . } 33.42 \% \\
& \mathrm{PbO} \text {. . } 52.65 \% \\
& \mathrm{~K}_{2} \mathrm{O} \cdot . \quad .12 .21 \% \\
& \mathrm{H}_{2} \mathrm{O} \text {. . } 1.64 \%
\end{aligned}
$$

Diese Zusammensetzung entspricht nicht völlig, aber sehr nahe der Formel $2 \mathrm{PbCrO}_{4} \cdot \mathrm{K}_{2} \mathrm{CrO}_{4} \cdot \mathrm{H}_{2} \mathrm{O}$.

Dasselbe Produkt entsteht, wenn man die Bleilösung mit dem gleichen Volum der Kaliumchromatlösung fällt und Niederschlag samt Mutterlauge bis zur Trockene verdampft.

Auch der zitronengelbe Niederschlag, der bei der Fällung der Kaliumchromatlösung mit dem halben Volumen der Bleilösung erhalten wird, hat nahezn dieselbe Zusammensetzung.

DaB bei der Fällung sehr konzentrierter Lösungen und Anwendung eines großen Überschusses von Kaliumchromat die Verbindung $\mathrm{PbCrO}_{4} \cdot \mathrm{K}_{2} \mathrm{CrO}_{4}$ entsteht wurde schon früher ${ }^{1}$ ) nachgewiesen.

1) Z. anorg. Chem. 54 (1907), 192.

z. anorg. u. allg. Chem. Bd. 109. 
Wird der ohne ChromatüberschuB gefällte hellgelbe amorphe Niederschlag mit der Mutterlauge erbitzt, so wird er orangegelb und kristallinisch, und nach dem Auswaschen mit kochendem Wasser hinterbleibt fast normales Bleichromat (bei der Analyse gefunden: $30.26 \% \mathrm{CrO}_{3}$ ).

Der mit geringem ChromatüberschuB gefällte schon kristallinisch gewordene Niederschlag gibt bei der gleichen Behandlung an die Waschwässer kleine Mengen Kaliumchromat ab und hinterläbt schlieblich normales Bleichromat (bei der Analyse gefunden: $31.01 \% \mathrm{CrO}_{3}$ ).

Während die bei der Fällung der Bleilösung mit Kaliumchromat ausfallenden Niederschläge kaliumhältig sind, sind dịe durch Natriumchromat gefällten natriumfrei.

Tropft man in 11 Volum der Bleilösung 10 Volum der Natriumchromatlösung, so entsteht wieder ein hellgelber Niederschlag, der unter dem Mikroskop das gleiche Aussehen zeigt, wie der durch Kaliumchromat erhaltene. Bei längerer Berührung mit der Mutterlauge bleibt er unverändert. Auch beim Wegwaschen der Natriumacetat und wenig Bleiacetat enthaltenden Mutterlauge mit Alkohol behält er seine hellgelbe Färbung und sein mikroskopisches Aussehen. Die Analyse eines so hergestellten und bei $120^{\circ}$ getrockneten Niederschlags ergab:

$$
\begin{aligned}
& \mathrm{CrO}_{3} \cdot . \quad 29.55 \% \\
& \mathrm{PbO} \text {. . } 69.31 \% \\
& \mathrm{H}_{2} \mathrm{O} \text {. . } \quad 0.92 \%
\end{aligned}
$$

Aus dem Molekularverhältnis $\mathrm{CrO}_{3}: \mathrm{PbO}=1: 1.052$, welches sich daraus berechnet ergibt sich, daB er ein schwach basisches Bleichromat ist. $\mathrm{DaB}$ er natriumfrei ausfällt ist jedenfalls dem Umstand zu rerdanken, daß bei der Einwirkung einer Lösung von Natriumacetat auf Bleichromat kein Austausch zwischen Natrium und Blei stattfindet, es geht kein Bleiacetat in Lösung.

Tropft man in 10 Volum der Bleilösung 11 Volum der Natriumchromatlösung, so entsteht zunächst wieder derselbe Niederschlag, bei längerer Berührung mit der Mutterlauge geht dieser aber allmählich in einen orangegelben kristallinischen Niederschlag über. Ein so hergestellter mit wenig kaltem Wasser von der anhaftenden Mutterlauge leicht befreiter Niederschlag, bei $120^{\circ}$ getrocknet, gab bei der Analyse:

$$
\begin{aligned}
& \mathrm{CrO}_{3} \cdot . \cdot 30.62 \% \\
& \mathrm{PbO} \cdot . \\
& \hline
\end{aligned}
$$

war somit normales Bleichromat. 
Auch bei der Fällung der Bleiacetatlösung mit Ammonium. chronat erhält man bei Anwendung eines Bleiüberschusses einen hellgelben amorphen, durch einen Chromatüberschuß orangegelb und kristallinisch werdenden Niederschlag, der unter dem Mikroskop das gleiche Aussehen zeigt, wie die mit Kalium- oder Natriumchromat gefällten Niederschläge.

Ein aus 11 Volum der Bleilösung und 10 Volum der Ammoniumchromatlösung erhaltener hellgelber Niederschlag, abfiltriert, durch Auswaschen mit Alkohol von der anhaftenden Mutterlauge befreit und bei $120^{\prime \prime}$ getrocknet, gab bei der Analyse:

$$
\begin{aligned}
& \mathrm{CrO}_{3} \text {. . } 29.83 \% \\
& \mathrm{PbO} \text {. . } 69.36 \% \\
& \left(\mathrm{NH}_{4}\right)_{2} \mathrm{O} . \quad 0.61 \%
\end{aligned}
$$

Daraus berechnet sich das Molekularverhältnis $\mathrm{CrO}_{3}: \mathrm{PbO}$ : $\left(\mathrm{NH}_{4}\right)_{2}=1: 1.042: 0.039$. Ist das Ammonium als normales Chromat im Niederschlag, so ist der damit verbundene Rest wieder ein schwach basisches Bleichromat mit dem Molekularverhältnis $\mathrm{CrO}_{33}$ : $\mathrm{PbO}=1: 1.083$.

Daß der Niederschlag ammoniumbältig ist, erklärt sich wieder dadurch, daB bei der Einwirkung einer Lösung von Ammoniumacetat auf Bleichromat ein geringer Austausch zwischen Ammonium und Blei stattfindet, es geht eine kleine Menge Bleiacetat in Lösung und Ammoniumchromat in den Niederschlag.

10 Volum der Bleilösung mit 11 Volumen der Ammoniumchromatlösung gefällt gaben zunächst denselben Niederschlag, bei längerer Berührung mit der Mutterlauge ging er aber völlig in einen orangegelben kristallinischen über. der beim Auswaschen mit kaltem Wasser kleine Mengen Ammoniumchromat an dieses abgab und reines normales Bleichromat hinterlieB. (Bei der Analyse gefunden: 30.99\% $\mathrm{CrO}_{3}$ ).

Wird der hellgelbe Niederschlag, der aus überschüssiger Bleilösung durch Natriumchromat gefällt wird, abfiltriert und mit kaltem Wasser ausgewaschen, so erhält man, auch nachdem der ÜberschuB der Bleilösung weggewaschen ist, farblose und klare Filtrate. Bei der Fällung mit Kaliumchromat hingegen kann man. nur durch Anwendung eines sehr dichten Filters (Blaubandfilter von ScHLErcher und SCEÜLL) und Vermeidung der Saugpumpe anfangs noch schwach getrübte, später aber klare Filtrate erhalten. Auch der durch Ammoniumchromat gefällte gibt beim Auswaschen, sobald der ÜberschuB der Bleilösung weggewaschen ist, trübe Filtrate. In allen 
Fällen wird bei andauerndem Waschen der Niederschlag auf dem Filter orangegelb und kristallinisch. Unter dem Mikroskop zeigt der mit Kaliumchromat erhaltene wieder dünne kurze Nädelchen, der mit Natrium- oder Ammoniumchromat erzielte eckige Körnchen. Die Analyse so hergestellter und bei $120^{\circ}$ getrockneter Niederschläge ergab:

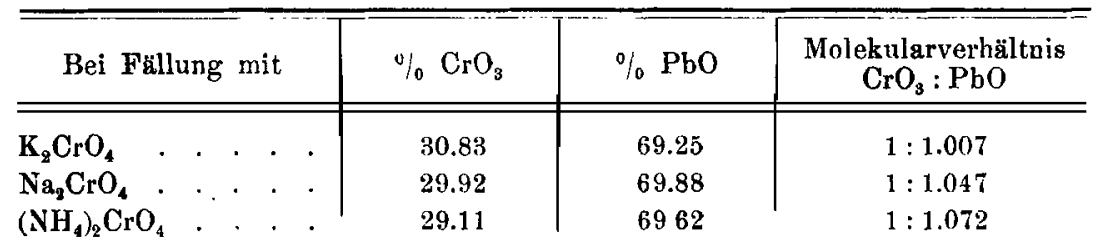

Danach ist das kristallinische Endprodukt der Einwirkung des Wassers auf dem kaliumlältigen Niederschlag ein normales, auf die beiden anderen ein schwach basisches Bleichromat, vermutlich weil bei letzteren der Chromsäuregehalt zar vollständigen Umwandlung des basischen in normales Chromat nicht hinreicht. Dadurch ist wahrscheinlich anch ihr von ersterem verschiedenes Aussehen unter dem Mikroskop zı erklären.

Ginge bei der Zersetzung des kaliumhaltigen hellgelben Bleichromats durch Wasser einfach nur Kaliumchromat in Lösung, so. müBte als Rückstand ein basisches Chromat verbleiben, daB dies nicht der Fall ist, erklärt sich dadurch, daB eine verdünnte Kaliumchromatlösung an Bleioxyd, sowie auch an basische Bleichromate Chromsäure unter gleichzeitiger Bildung von Kaliumbydroxyd abgibt.

Die Bildung einer feinen Suspension, die beim Auswaschen der Niederschläge mit kaltem Wasser durch das Filter läuft, konnte nur bei den kalium- oder ammoniumbältigen beobachtet werden. Daraus ist zu schließen, daß dieses feinverteilte Bleichromat von der Zersetzung von Kalium- oder Ammoniumbleichromat durch Wasser herrührt. Filtriert man einen aus $5.5 \mathrm{ccm}$ der Bleilösung und $5 \mathrm{ccm}$ der Natriumchromatlösung erhaltenen Niederschlag, der solche Doppeichromate nicht enthält auf einem gewöhnlichen Papierfilter $a b$ und wäscht ihn mit kaltem Wasser rasch aus, so erhält man vollkommen farblose klare Filtrate. Gießt man dann aber eine Kaliumchromatlösung auf das Filter und wäscht, wenn diese abgelaufen ist, abermals mit Wasser aus, so erhält man sofort wieder durch einen feinen gelben Niederschlag getrübte Filtrate. Eine kolloide Lösung entsteht hierbei nicht, denn der trübende Nieder- 
schlag setzt sich bei ruhigem Stehen der Flüssigkeit zwar sehr langsam, aber vollständig $a b$.

Das für die hellgelben amorphen Niederschläge gefundene Molekularverhältnis $\mathrm{CrO}_{3}: \mathrm{PbO}=1: 1.052$ bis 1.104 , welches von dem Verhältnis 1:1 nur wenig abweicht und ihr gleichartiges Aussehen unter dem Mikroskop lassen vermuten, dab sie im wesentlichen aus amorphen Bleichromat bestehen, für dessen Bestand ein Bleigehalt der Lösung mit der es in Berührụng steht Bedingung ist, der aber gleichzeitig den Mehrgehalt an $\mathrm{PbO}$ im Niederschlag verursacht. Dieser ist wahrscheinlich auf Adsorption basischen Bleichromats zurückzuführen.

Die Schutzwirkung von Bleisalzlösungen gegen den Farbenumschlag von hellgelb in orange bei der technischen Darstellung der hellen Chromgelbe hat schon JABLCzYNSKI ${ }^{2}$ ) untersucht, und auf Grund der Ionentheorie eine Erklärung hierfür gegeben. Danach wäre dieser Farbenumschlag einer geringen Beimengung roten basischen Bleichromats zu hellgelbem normalem zuzuschreiben. Auch nach $Z_{E R R}{ }^{2}$ ) ist die Veränderlichkeit der Färbung der Chromgelbe beim Trocknen auf eine Bildung dunkler gefärbter basischer. Salze zurückzuf ühren.

Nach den oben angeführten Analysen sind aber gerade die aus wässeriger Bleiacetatlösung bei gewöhnlicher Temperatur gefällten hellgelben Niederschläge stärker basisch als die dunkleren und der Farbenumschlag scheint nur durch den Übergang eines hellgelben amorphen in ein orangegelbes kristallinisches Bleichromat bewirkt zu werden. Das ist vielleicht so zu erklären, daß die feinen Teilchen des amorphen Bleichromats in Berührung mit der Bleiacetatlösung sich an der Oberfläche mit einer dünnen Schicht basischen Chromats bedecken, welche die Kristallisation verhindert, während eine Alkalichromatlösung durch Abgabe von Chromsäure an das basische Salz dieses Hindernis beseitigt und dadurch die Kristallisation einleitet.

Die verhältnismäBig kleinen Mengen Kaliumchromat (5.92\%) und Ammoniumchromat $(1.78 \%)$, welche in den hellgelben Niederschlägen gefunden wurden, siud wahrscheinlich als Kalium- ${ }^{3}$ ) oder Ammoniumbleichromat ${ }^{4}$ ) der überwiegenden Menge freien Bleichromats beigemengt.

1) Chem. Zentralblatt 1909, I, 728.

2) Chem. Zentralblatt 1912, I, 1344.

3) Z. anorg. u. allg. Chem. 54 (1907), 192.

4) Z. anorg. w. allg. Chem. 58 (1908), 424. 
Die im vorstehenden gegebenen Beleganalysen wurden folgendermaBen durchgeführt:

Der Chromsäuregehalt wurde nach dem eingangs erwähnten jodometrischen Verfahren bestimmt.

Zur Bleibestimmung wurden die Proben in einem gerïumigen durch ein Uhrglas lose bedeckten Kolben mit mäßig konzentrierter Salpetersäure und wenig Alkohol so lange erhitzt bis sie ganz in Lösung gegangen waren und keine nitrosen Dämpfe mehr entwichen. Dann wurde die Lösung in einer Porzellanschale auf dem Wasserbade mit einem ÜberschuB verdünnter Schwefelsäure bis zur Trockene verdampft, der Trockenrückstand mit verdünnter Schwefelsäure aufgenommen und das ungelöst gebliebene Bleisulfat im Goocu-Tiegel gesammelt, erst mit verdünnter Schwefelsäure, dann mit Alkohol gewascheu, scharf getrocknet und gewogen.

Zur Bestimmung des Kaliums wurden die Proben durch Erhitzen mit konzentrierter Salzsäure thd darauffolgendem Zusatz von viel heiBem Wasser in Lösung gebracht. Dann wurde das Blei durch Schwefelwasserstoff, das Chrom durch Ammoniak ausgefällt, das Filtrat von den entsprechenden Niederschlägen in der Platinschale eingedampft, Ammoniumchlorid abgeraucht und das zurückgebliebene Kaliumchlorid gewogen.

Zur Bestimmung des Ammoniums wurde aus den Proben durch Lrhitzen mit Kalilauge Ammoniak ausgetrieben, in einer gemessenen Menge titrierter Salzsäure aufgefangen und der verbliebene Säureüberschuß zurückgemessen.

Der Wassergehalt wurde aus dem Gewichtsverlust berechnet, welchen die bei $120^{\circ}$ getrockneten Proben durch Erhitzen auf dunkle Rotglut erlitten, bei welcher Temperatur Bleichromat, falls nicht gleichzeitig reduzierend wirkende Stoffe zugegen sind, noch keinen Sauerstoff abgibt.

Wien, Chemisches Laboratorium der Staatsgewer-beschule.

Bei der Redaktion eingegangen am 10. Oktober 1419 . 